\title{
Strategies for Context-Adaptive Message Dissemination in Vehicular Ad Hoc Networks
}

\author{
Stephan Eichler*, Christoph Schroth*, Timo Kosch ${ }^{\dagger}$, and Markus Strassberger ${ }^{\dagger}$ \\ ${ }^{*}$ Institute of Communication Networks \\ Technische Universität München, 80290 Munich, Germany \\ Email: s.eichler@tum.de, christoph.schroth@cdtm.de \\ ${ }^{\dagger}$ BMW Group Research and Technology \\ Email: timo.kosch@bmw.de, markus.strassberger@bmw.de
}

\begin{abstract}
In future deployments of Vehicular Ad hoc Networks (VANETs) safety-related applications such as local danger warning (LDW) will use broadcast-based communication schemes to transmit information to other vehicles within the network. The unlimited flooding of a message throughout the whole network however, is neither feasible nor intended. The high resulting traffic load would congest the shared wireless medium and prevent other, potentially highly relevant and time-critical messages from getting access to the medium. Existing strategies to limit redundant packet retransmissions manage to make broadcast-based data packet dissemination more efficient and reliable. However, they do not take into account the individual network's nodes interest in information. In fact, the static mechanisms proposed are not adequate to leverage the limited network resources as efficiently as possible in varying network conditions and to transport information to where it is needed as fast as possible. Therefore, we propose an altruistic communication scheme which differentiates data traffic according to the benefit it is likely to provide to potential recipients. A system for calculating and leveraging message benefit and two different node architectures are presented. With the help of a comprehensive simulation environment, the performance of our concept is analyzed. Comparative simulative studies show that an improvement of the benefit provided to all the participants in a VANET is also possible with the help of the readily available IEEE 802.11e standard, but to a lower extent.
\end{abstract}

\section{INTRODUCTION}

Inter-vehicular communication has been identified to bear great potential to improve both road traffic safety and comfort. Possible use-cases reach from safety-related warning systems to amended navigation mechanisms and entertainment applications. The so called Local Danger Warning systems [1] are within the focus of this work. Enormous challenges are inherent to the deployment of such applications in a VANET environment. Conventional unicast communication schemes, where one network node exchanges data with one other node, are not appropriate: The high mobility of the vehicles and the resulting, frequent network topology changes challenge existing routing protocols, which quickly suffer from outdated neighborhood and routing data. Data is spread among the wireless enabled vehicles in a broadcast fashion. The most intuitive propagation methodology is often referred to as "Flooding", where every network node re-broadcasts each single packet it receives. The main problem inherent to this technique is the huge amount of superfluous retransmissions that absorb the limited network resources and possibly prevent highly relevant data from being transmitted. Simple dissemination limitation strategies such as Geocast [2] work as a first approach to reduce channel congestion, but do not account for the individual nodes' interest nor are they able to optimally leverage network resources in both sparse and dense networks. Other approaches of intelligent restrictions to a mere flooding as presented in [3]-[5] help reducing redundant retransmissions and making a broadcast-based propagation of messages more reliable and efficient. However, they cannot ensure that information is transported to where it is needed with an as small as possible latency while optimally leveraging the available network resources.

Context is an important issue for warning applications in VANETs: A message containing a traffic congestion warning for the city center will most likely be of no use for the driver of a vehicle on the highway around the city. However, the driver of a vehicle on the same highway entering the city might be interested in the message. Therefore, information on e.g. location, direction, speed, and driver preferences are important parameters influencing the value of warning messages. In this paper we propose an approach that is able to take into account individual drivers' interest for determining message importance and benefit. We pass on pre-determined, static propagation limits in either the time or the local domain and leverage a decentrally managed, situation-adaptive content evaluation and dissemination strategy. Thereby, network nodes apply a mechanism to evaluate the benefit messages may provide to potential recipients and prioritize data packets accordingly. For example, if the network load is high, but connection time between two vehicles is very short, the most relevant data is broadcast first.

The remainder of the paper is organized as follows. Sec. II provides an overview of existing strategies to cope with the challenges mentioned above. Sec. III proposes methods to evaluate message benefit and to leverage the resulting values in different node architectures. Sec. IV] introduces a comprehensive simulation environment and shows various results. Sec.V concludes with a short summary. 


\section{RELATED WORK}

As mentioned in the introductory part, the overall goal of this paper is to elaborate on a communication protocol which allows for benefit-based message dissemination in VANETs. For this reason, extensive research has been performed mainly regarding three different topics: First, numerous definitions of the benefit provided by traffic related information have been found and are briefly outlined in the following section. This section also features a brief overview of different methods in the field of Network Utility Maximization (NUM). Second, we need to clearly understand existing approaches to making broadcast-based message propagation in VANETs both reliable and efficient. Diverse techniques are presented in the following and serve as a basis for the design of our proprietary communication protocol presented in Sec. III. Last, differentiation of data traffic turned out to be a crucial, integral part of our protocol as well. Therefore, different ways of introducing such a differentiation are presented.

\section{A. Quantifying the Benefit of Traffic Related Information}

Numerous approaches to defining and quantifying the benefit (often also referred to as utility) of traffic related information can be found in literature:

In [6], an overview of the different existing kinds and categories of traffic information is presented. A framework is set up for understanding the effects of such information not only on road users and thus on traffic, but also on various other stakeholders. As possibility for quantifying potential positive and negative impacts, the authors propose measures such as reduced number of accidents, reduced environmental pollution or shorter travel times. However, these dimensions can neither be objectively compared with each other nor computed or observed before vehicles have made decisions on the basis of traffic information. When thinking of an efficient, benefit-based message dissemination algorithm, however, a first estimate on the benefit provided by a certain message must be possible exactly at the point in time before sending the message.

Finally, numerous publications deal with the topic of improving the usefulness of mobile ad hoc networks in general, i.e. without a focus on traffic related information. As it is the goal of this paper to identify possibilities to maximize the benefit (also referred to as utility) all network participants receive, we also present some major approaches dealing with the so called Network Utility Maximization (NUM) problem. [7], for example, addresses the difficulties in designing universally valid communication protocols that are adequate over all different deployment scenarios and environments and introduces the term utility. In this context, the authors define utility as the degree of fulfillment of application-specific requirements. A Voice-over-IP application, for example, is only regarded to be useful if certain packet delay and jitter requirements are met by the network. So called utility functions must be set up to reflect all the application-dependent utility criteria and have to be composed into one mathematical framework which can then be used to maximize the aggregate system utility, i.e. the utility all

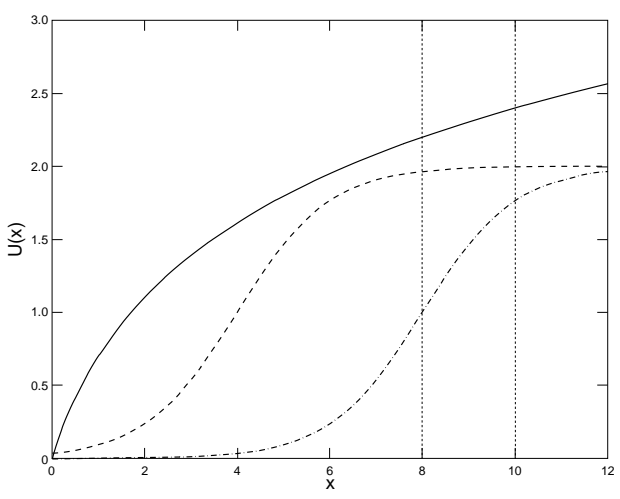

Fig. 1. Concave and sigmoid examples of utility functions as shown in [11]

the network nodes experience. Since the publication of [8], the maximization of the so called global utility has also become an important principle underlying several rate allocation schemes and congestion control protocols for diverse communication networks. In [9], the basic approach to realize utility-based data rate control and thereby maximize the aggregate, global network utility can be studied in detail:

Each of the $K$ existing network users $i$ utilizes a certain set of links (also referred to as resources) and is provided an individual data rate $x_{i}$. The utility a certain user experiences solely depends on this data rate he has available for his application, so $U_{i}\left(x_{i}\right)$ represents the utility function. In their model, the authors assume increasing, strictly concave and continuously differentiable functions of $x_{i}$, where $x_{i} \geq 0$. Examples for both concave and sigmoid utility functions can be seen in Fig. 11 Matrix $A$ defines fixed routes within the network, whereas $C$ represents the resources' maximum capacities. The goal of this approach is to calculate data rates $x_{i}$ for each of the users on the basis of the available network resources and to show that the global, aggregate utility is maximized (Eqn. 11). The first constraint (Eqn. 2) says that the total data rate of a link may not be larger than its capacity. Assuming that utility functions are known, this problem can be solved mathematically. The authors, however, thereby assume that a central instance is existing that knows all the individual utility functions. The central coordinator can then allocate data rates to the single users such that the overall aggregate utility is maximized. [10] and [9] propose extensions that also work on a completely decentral basis, where nodes do not need any direct feedback from the network to be operational.

$$
\begin{array}{r}
\text { maximize } \sum_{i \in K} U_{i}\left(x_{i}\right) \\
\text { subjectto } A^{T} x \leq C \\
\text { over } x \geq 0
\end{array}
$$

Among the major weaknesses of the presented NUM algorithms with respect to our requirements are: Most of the schemes assume the existence of certain utility functions, but do not elaborate on a comprehensive model of user needs and 


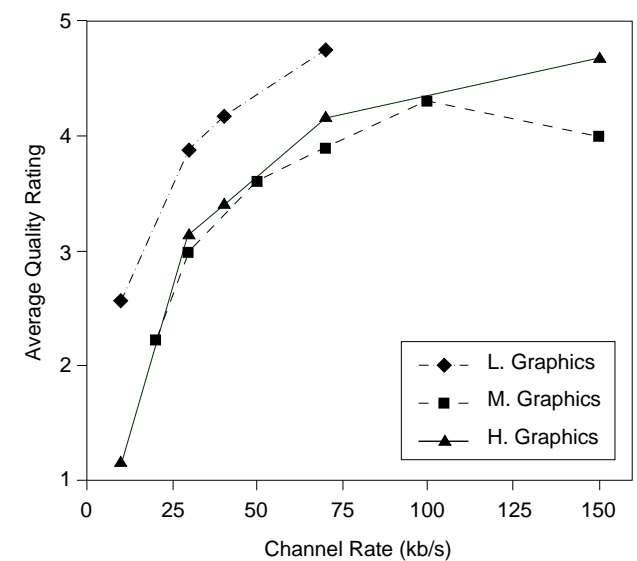

Fig. 2. Survey results: Concave utility functions for a web browsing application [12]

their perception of utility provided by different applications. However, utility provided to users in most cases cannot be quantified with the help of simple convex or concave functions which only depend on measures such as packet delay, throughput and jitter. Apart from that, NUM schemes head for a globally fair allocation of resources to the different network participants. Our approach for modifying resource allocation foresees the realization of controlled unfairness, i.e. stations are not granted access to the medium on an equal basis, but depending on the benefit of their available information.

A study focusing on the determination of utility functions can be found in [12]. The authors quantitatively (on a scale between 1 and 5) investigate subjective user experiences in different network conditions and thereby try to establish a comprehensive set of utility graphs. These graphs relate the service delivered by the network (in terms of throughput, delay, etc.) to user's satisfaction for different applications. Fig. 2 shows a first example of a translation of "hard" performance metrics to actual user quality rating. The link speed is varied between 10 and $150 \frac{\mathrm{Kb}}{\mathrm{s}}$, where the websites tested are divided into three categories (low graphics, medium graphics and high graphics). As one would have expected, as the available data rates increase, the average quality ratings improve and then start to flatten out as the rate continues to increase. In the context of traffic related data dissemination, car manufacturers will also have to set up similar field tests with a significant amount of test drivers to find out about the experienced benefit of various messages. However, to allow for first computational simulations, a model comprising application-specific benefit functions has been set up in the frame of this work, based on road traffic statistics and other empirical observations.

\section{B. Snapshot of Existing Strategies to Improve Reliability and Efficiency for Broadcast-based Message Dissemination}

[5] provides a comprehensive overview of the numerous algorithms which all basically aim at minimizing the number of redundant re-broadcasts when applying broadcast-based communication schemes and to make sure packets are reliably propagated to all desired destination nodes. In [13], Chiasserini et al. analyze the implications of adapting the magnitude of the so called contention windows to the distance between sender and receiver of a certain message. By introducing such a spatial traffic differentiation, the efficiency of broadcast-based message dissemination schemes can be improved. The greater the distance between the sending node and the recipient, the smaller the contention window is adjusted. Thereby, the likelihood that nodes which reach a maximum number of new neighbors can be maximized, where retransmissions conducted by close-by nodes (which would only reach a small amount of new receivers) are reduced to a minimum. In the frame of the contention-based forwarding technique [14], Füßler et al. present an algorithm which helps efficiently delivering messages to certain geographic regions while using broadcast communication. In contrast to conventional methodologies, not the source of a packet determines the next forwarder, but all neighbors of that source node are included in the decision making process of finding the most suitable next sender. Timers are chosen to realize a decentrally organized contention between forwarding candidates, and suppression strategies are proposed to avoid redundant retransmissions.

These algorithms help reducing redundant transmissions especially in dense networks. However, the specific content of the messages disseminated is not taken into account at all when deciding upon forwarding a message or not. For example, a local danger warning generated miles away from the receiver is treated exactly the same way as one created by the direct network neighbor (which intuitively is of a higher relevance for the recipient). To wind up with a scalable message dissemination approach especially for scenarios with limited resources, messages should be prioritized with regard to the local benefit they may provide for their recipients.

\section{Data Traffic Differentiation in VANETs}

Some publications already highlighted the necessity to treat data traffic in VANETs according to its respective relevance. For example in [15] the authors investigate the effects of a prioritized medium access scheme in the context of highly loaded VANETs. The authors introduce an implementation of the Enhanced Distributed Channel Access (EDCA) functionality which is the basis of the IEEE 802.11e standard [16]. Simulations show that in highly loaded network scenarios, the reception probability of packets can be increased by modifying parameters defining the medium access functionality.

Gerharz et al. present in [17] a token balancing scheme similar to other fair queuing strategies. Data packets are classified into two service classes and buffered in two different queues. Queues have to trade in tokens to get channel access. The more tokens a queue owns the higher is the access probability.

\section{A GENERAL FRAMEWORK FOR BENEFIT-ORIENTED INFORMATION DISSEMINATION}

Two fundamental methodologies form the basis of our novel approach: First, nodes have to quantify the benefit their respec- 
tive data packets provide to potential recipients in the local neighborhood. Second, message broadcast must be prioritized according to the resulting benefit values in order to maximize the benefit received by all the vehicles participating in the network (global benefit). Before elaborating on possibilities of quantifying message benefit and presenting diverse mechanisms for realizing a communication protocol for optimizing the global benefit, the major qualitative characteristics of our concept must be argued:

Altruism: In our concept, altruism is the major overall characteristic. Nodes do not primarily aim at maximizing their own benefit, but head for transmitting information such that their neighbors are provided with the data they are most interested in. Before transmitting a data packet, each node evaluates the benefit of all of its currently available messages and broadcasts the one currently providing the most benefit to its neighbors.

Application-oriented information differentiation: Existing approaches to improve the performance of message dissemination in mobile ad-hoc networks as introduced in Sec. II mostly rely on packet-specific data such as the geographical location of the last forwarder or the time at which the packet was generated. We, however, take into account applicationlevel data to enable a benefit-evaluation of single messages.

Decentrally controlled message scheduling: Our concept does not comprise one single, central schedule for packet transmission. Neither does it intend to decentrally realize a globally uniform schedule such as presented in [18]. In fact, each vehicle autonomously evaluates the benefit certain data may provide to potential recipients only based on its local and necessarily limited knowledge.

Sustainability through flexibility: Our concept provides full flexibility to changing requirements and will work both in dense and sparse networks. Due to non-static transmission restrictions, network resources can be optimally leveraged in different network environments. "Hard" limitations in both the local and time domain are avoided: Messages are relayed anyway, where traffic prioritization ensures that low-priority messages do not absorb the wireless medium and prevent highpriority messages from being transmitted. Thus, our approach is appropriate both for initial VANET deployments, but also for future, high-density scenarios.

Highly Integrating approach: To make broadcast communication reliable, efficient and to guarantee an optimal information dissemination, there are many specific approaches existing which all tackle part of the challenges inherent to VANETs. Our concept may work as a comprehensive substitute for numerous of these approaches. As will be shown in future work, our concept integrates ideas such as those incorporated in the contention-based forwarding approach [14] or those underlying the DPS [18] methodology.

\section{A. Quantification of Traffic Information Benefit}

As introduced in Sec. II various possibilities to quantifying the benefit of traffic information with the help of measures such as reduced number of accidents, reduced environmental

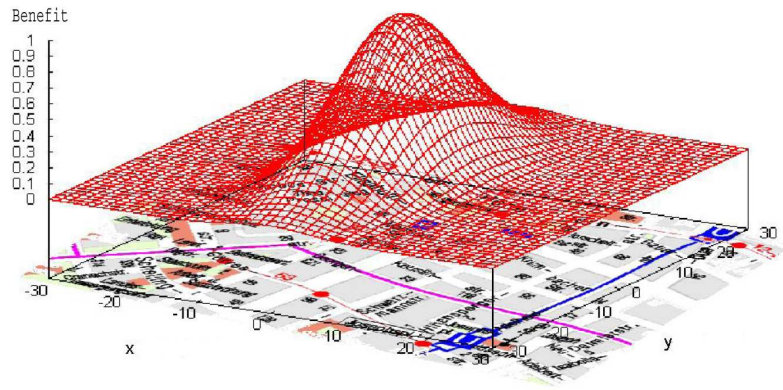

Fig. 3. Exemplary benefit sub-function for computing the parameter "distance to information source" as presented in [1]

pollution or shorter travel times [6] are existing. These dimensions are not adequate for a benefit approximation exactly at the point in time when receiving the message.

This, however, can be realized by taking the following context information into account for the benefit calculation:

1) Message context $m$ : is characterized by e.g. the message age, time since last broadcast, and time since last reception of a message.

2) Vehicle context $v$ : is described by e.g. the driving direction, distance to the last forwarder, current number of reachable neighbor nodes, distance to adjacent nodes and their respective driving directions, route flexibility, vehicle speed, and current road type.

3) Information context $i$ : is specified by e.g. the time of day, purpose of traveling, distance to the source, information accuracy, information category, news value, and change rate of the information.

A model comprizing sub-functions for each of these parameters and weights for combining the results of these subfunctions has been setup in the frame of this work, based on road traffic statistics and other empirical observations. Eqn. 4 highlights the way the benefit of a specific piece of information can be quantified.

$$
\text { MessageBenefit }=\frac{1}{\sum_{i=1}^{N} a_{i}} \cdot \sum_{i=1}^{N} a_{i} \cdot b_{i}(m, v, i)
$$

Supposed there are $N$ parameters such as distance to information source and message age. They all have to be computed with the help of the message content $m$, the vehicle context $v$ and the information context $i$. All these $N$ parameters are evaluated with application-dependent sub-functions, which result in the values $b_{i}$. The resulting parameters finally have to be weighted with application-dependent factors $a_{i}$. In the context of a crossing collision warning, for example, the message age parameter is more important for the overall message benefit than the parameter evaluating the information quality. Last, all weighted parameters have to be summed up and divided by the sum of the weighting factors.

An important assumption underlying this altruistic approach of differentiating traffic according to its respective benefit 
for neighbor nodes is that adjacent nodes evaluate certain messages in a similar way, i.e. the benefit values computed by sender and receiver of the same message must be similar. Provided that this postulate holds, a different prioritization of data helps fulfilling the requirements of a scalable and efficient communication protocol for broadcast-based VANETs.

In our simulative model, each vehicle maintains a so called benefit account which is used to determine the overall benefit within the scenario. Upon message reception, the application immediately evaluates the information with the help of the abovementioned benefit function. The resulting value can vary between 0 (no benefit) and 1 (high benefit). This value is then added to the account which represents the overall benefit received from all previous messages. We refer to the sum of all benefit accounts of all vehicles at a certain point in time as the global benefit, which is our equivalent to the overall network utility presented in Sec. III. It represents the benefit provided to all wireless enabled cars within a scenario and is the measure to be maximized with the help of our novel message dissemination strategy.

\section{B. Protocol Modifications to Enable Benefit-Based Traffic Differentiation}

In our model, vehicles are assumed to initiate message transmissions in three different situations: First, cars may detect a local danger, thereupon generate a proper warning message and broadcast it. Second, messages may be received and can immediately be relayed to the respective neighbors. Third, vehicles broadcast all their stored messages if a previously unknown communication partner is detected with the help of periodically sent beacon messages. Before finally transmitting any data packet, each vehicle independently evaluates the current benefit of the packets and applies the following three levers, which are part of the application layer and the data link layer.

First, a variable benefit threshold is introduced. When active, only messages providing more benefit than the pre-determined threshold are broadcasted, relayed, and stored in the nodes' local memories. Thus, actually redundant broadcasts can be avoided and the channel is kept free for more relevant messages.

Second, an in-vehicle packet scheduling has to ensure that messages are broadcasted in a sequence established according to their respective benefit values. All packets a node intends to transmit are passed down from the application layer to the MAC layer and are first of all buffered in the so called interface queue. The packet dequeuing procedure does not work according to the conventional FIFO principle any more. Instead, it takes into account the benefit packets may provide when deciding which packet is to be dequeued next. An additional, optional packet enqueuing amendment works as follows: Not necessarily the newly arriving packet, but the one with the lowest benefit value in that moment is discarded in case of a queue overflow.

Third and last, the inter-vehicle contention for the shared medium must be adapted such that the most relevant packet all nodes within mutual communication range have available is granted access to the medium. For this purpose, the dynamic adaptation of the so called contention window (CW) within the MAC layer is used as the main influence on medium access. Each station uses its individual $\mathrm{CW}$ to select both backoff and defer timers, which represent a key feature of the CSMA mechanism.

By selecting short contention windows in case a highly relevant packet requests access to the medium, its likelihood to win the contention process is increased. The smaller the CW, the shorter the timers defining the period of time after a node may try or retry to access the shared medium. Eqn. 5] shows the modified, benefit-based computation of a backoff timer, whose starting integer value is randomly determined from a uniform distribution over the interval $[0, C W]$. The slottime's length is set to $16 \mu$. $R$ is a random number and $C W_{b b}$ represents the node's current benefit based $\mathrm{CW}$. The calculation of $C W_{b b}$ is conducted according to Eqn. 6, where $C W_{\max }$ (1023 slots) and $C W_{\min }$ (31 slots) are the minimum and maximum CWs. Thus, the CW a node applies can always be adapted to the benefit (MessageBenefit $\varepsilon[0,1]$ ) of the currently handled message.

$$
\begin{aligned}
\text { BTimer }= & \left(R \bmod \left(C W_{b b}+1\right)\right) \cdot \text { SlotTime } \\
C W_{b b}= & ((1-\text { MessageBenefit }) \cdot \\
& \left.\cdot\left(C W_{\max }-C W_{\min }\right)\right)+C W_{\min }
\end{aligned}
$$

\section{Communication Protocol Architectures}

The cross-layer design depicted in Fig. 4 implements the three levers described above: The application layer is devoted to generating, broadcasting, receiving and storing messages. Since all application-level information such as geographic position and system time is available within this layer, it also implements the benefit function for evaluating the benefit of all data packets before passing them to the MAC layer. The resulting benefit values are included in each message's header. The introduction of the application layer into the communication protocol design is crucial since a comprehensive evaluation of message benefit can only be conducted in case message-, vehicle-, and information contexts are available.

The so-called benefit-based extension $(B B E)$ within the data link layer accounts for changing the functionality of the interface queue (modified enqueuing and dequeuing behavior) and the medium access control mechanism as described above. The extension may thereby leverage the values attached to the packets' headers or continuously re-evaluate the benefit of all enqueued packets with the help of the aforementioned benefit function. The inter-layer communication module (ILC) is used by the $B B E$ as means for acquiring all necessary, mostly application-level data to calculate up-to-date benefit values for all packets waiting for medium access.

One advantageous alternative to our cross-layer design would be to deploy the already existing IEEE 802.11e standard [16] for realizing a benefit-oriented traffic differentiation. The standard supports different so called traffic categories 


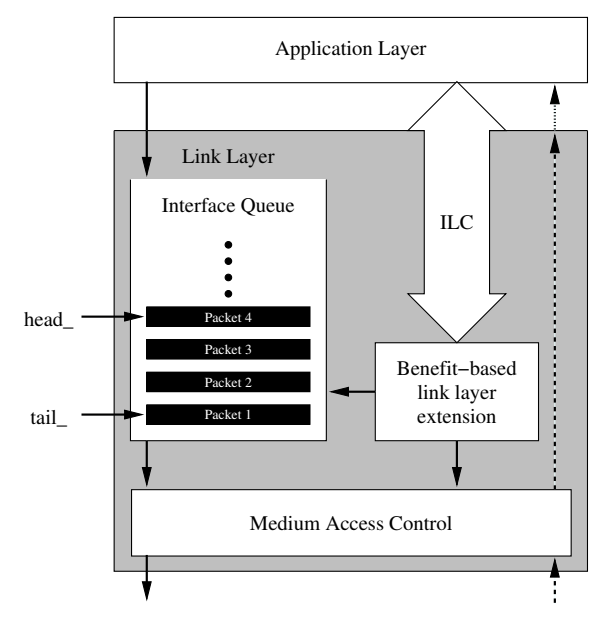

Fig. 4. Cross-layer architecture

(realized with the help of up to eight packet queues), of which each is assigned a certain priority determining the degree of favoritism with respect to access to the shared, wireless medium. Nodes applying the EDCA scheme conduct a two-step contention process: First of all, its different queues participate in an internal contention process which is conducted as if nodes contended for the real medium. As a consequence, virtual collisions occur frequently between the queues, upon which the higher priority packet is granted the chance to participate in the external contention process, where the colliding lower priority packet has to go into backoff. After doing so, the packet coming out ahead has to contend with packets from other stations for the real shared medium. This process is executed using the same parameters as before. The three main parameters which may be used to realize traffic differentiation are the minimum and maximum contention window $\left(C W_{\min }, C W_{\max }\right)$, the arbitration interframe space (AIFS), and the so called persistence factor $(P F)$. Low values for $C W_{\min } / C W_{\max }$, AIFS and $P F$ provide packets with a higher chance to win contention processes and to get quicker access to the medium. By evaluating message benefit within the application layer, attaching these values to the packet headers and sorting them into the proper priority queues, a prioritization of highly relevant traffic is possible as well. Packets with benefit values between 1 and 0.75 are sorted into the first priority queue, packets between 0.75 and 0.5 into the second queue and so on. Both internal contention between the packet queues and external contention for the real, wireless, shared medium support the transmission of the currently most relevant messages and thus are assumed to contribute to an improved global benefit.

\section{Evaluation by Simulation}

\section{A. Implementation of a Comprehensive Simulation Environ- ment}

As a basis for the computational analysis of our communication schemes, we used the ns2 simulator [19]. The implementation of the legacy IEEE 802.11 standard has been used as a basis for the representation of the link layer functionality in the ns2 environment. In terms of modeling radio wave propagation, we chose the Two Ray Ground Model [20], where the radio channel is assumed to be error-free. To simulate node mobility, the so called CARISMA traffic simulator, a proprietary tool [21], has been used. CARISMA uses ESRIShapefile road map data to position network nodes on a real street map (in this case Munich downtown, Germany). Vehicle positions and directions are updated on a timediscrete basis. The underlying mobility model follows the so-called Krauß approach [22] and thus allows for a very realistic vehicle movement representation. A more detailed description of the simulation environment has been presented in [23]. The application layer features a so called ad hoc agent that manages the data traffic and comprises the benefit function introduced before. The resulting values are attached to each of the packets' header before passing them down to the link layer. In the case of the data link layer, either the conventionally used and well verified representation of the IEEE 802.11 specification or an implementation of the IEEE 802.11e [16] standard (designed and deeply verified by Wiethölter et al. [24]) can be chosen. As described in Sec. IIIC. the 802.11e implementation ensures that each node operates four packet queues of which each applies different parameters regarding the medium access control functionality.

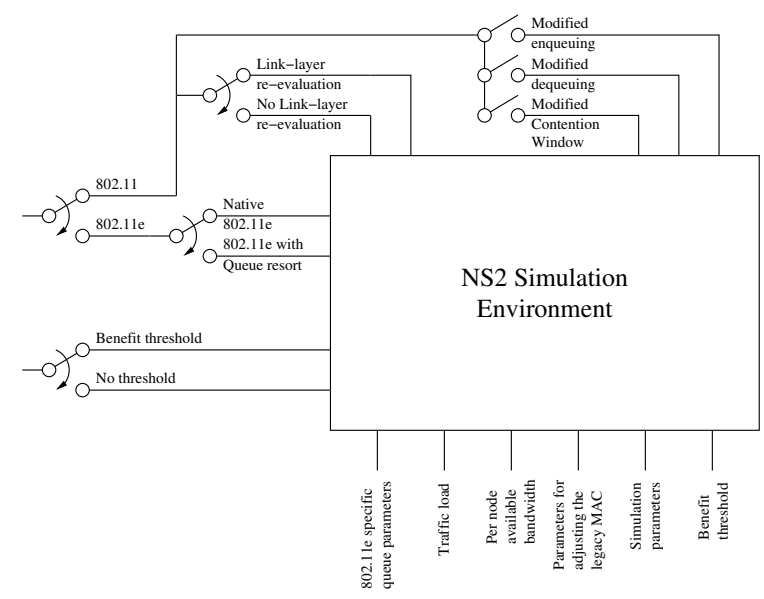

Fig. 5. Ns2-based simulation environment

\section{B. Simulation Runs and Results}

Numerous simulations have been conducted to analyze the performance of the mechanisms proposed above. The scenario which has been used for all of them is as follows: Within an area of $8 \mathrm{~km}^{2}, 300$ wireless enabled vehicles (all of which are assumed to be able to detect potential hazards) are moving around, where a communication range of $400 \mathrm{~m}$ has been applied, which was found to be rather realistic in field tests conducted by BMW Group Research and Technology. The simulation duration was set to $100 \mathrm{~s}$. For every scenario 50 independent iterations have been conducted to avoid statistical aberrations. Interface queues by default can store up to 200 


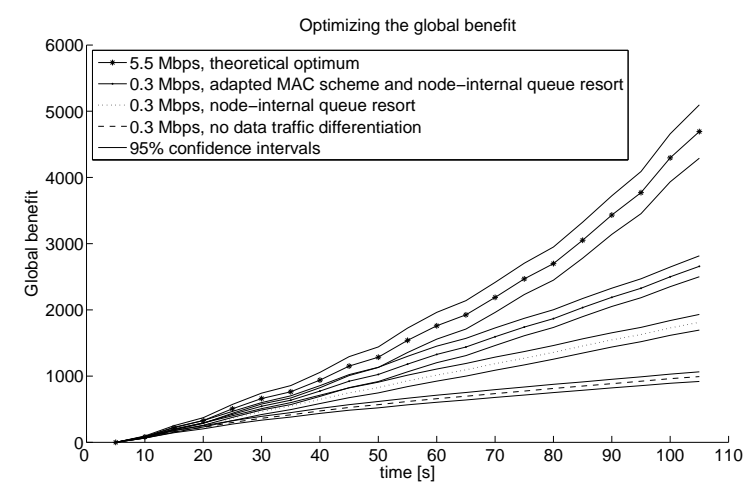

Fig. 6. Benefit improvement potential due to in-vehicle and inter-vehicle message selection

data packets. Ten different applications (ranging from crossing collision warning to congestion warning) are assumed to be run on each of the vehicles. To simulate highly loaded network scenarios, the nodes can only access a bandwidth between $0.1 \frac{\mathrm{Mbit}}{\mathrm{s}}$ and $0.5 \frac{\mathrm{Mbit}}{\mathrm{s}}$. This trick enables the simulation of highly loaded, large networks, while reducing the number of events in the simulator to a reasonable extent. The benefit function applied in the following scenarios comprises five different parameters: Distance from information source, message age, time since last reception, time since last broadcast, and general application relevance. As simulations with other functions and different parametrization showed, the improvement potential achieved due to the use of this specific function is valid without loss of generality.

1) Simulations using the cross-layer design: In Fig. 6 the results for a scenario using the legacy 802.11 MAC and a packet generation rate of 10 packets per second can be seen. Graph 4 shows the development of the global benefit, i.e. the sum of the local benefit accounts of all vehicles driving through the scenario, over time. A simple, packet benefit agnostic store-and-forward scheme is applied such that nodes try to broadcast all packets contained in their send buffers in the order they were passed down from the application layer. The interface queue simply works according to the FIFOprinciple. Graph 3, in contrast, shows that by only changing the internal broadcast sequence in a high-load scenario a huge improvement of the benefit provided to all vehicles can be achieved. By adapting the nodes' contention windows according to approximated benefit values, the global benefit can be improved even more (Graph 2). Due to the MAC adaptation as described above, channel utilization is slightly deteriorated due the prolonged defer- and backoff timers (we simulated a $3 \%$ drop of net data throughput). However, this throughput drop is compensated by the benefits of the inter-vehicle message selection by far. Graph 1 visualizes the theoretical benefit optimum: In case each node has a bandwidth of $5.5 \mathrm{Mbps}$ available, almost every packet can be sent immediately, queues do not emerge at all. As a consequence, all the proposed mechanisms do not show any effect.
An important assumption made in the context of our altruistic concept is that network nodes are able to properly estimate the benefit messages will provide for adjacent nodes. The great challenge inherent to our approach is to estimate the extent of interest all the neighboring vehicles have in a specific piece of information. Due to the autonomous benefit evaluation scheme, which does not foresee any context translation from one vehicle to the other prior to a message broadcast, individual vehicles never have explicit information available about what their neighbors are most interested in. In order to examine to which extent the benefit estimations are correct, the following analysis has been performed: Network nodes receiving a message read out the "Last benefit" field from the data packet and subtract it from the benefit value calculated on their own (based on their exact interest on information). The absolute value of the resulting difference represents the degree of estimation defectiveness and is visualized in Fig. 7. Note that in this case, the benefit function presented above was used. For a more clear appearance, the defectiveness intervals are labeled with numbers: 1 symbolizes a benefit value difference of $0-0.05$, where interval 2 is the range between 0.05 and 0.1 and so on. A benefit value difference of 1 would be the worst case (e.g., the sender regards a message as completely useless, where the receiver is extremely interested in it). As one can see in Fig. 7, in more than $55 \%$ of all received messages in this scenario, the difference between the sender's benefit estimation and the receiver's actual calculation was lower than 0.05. Slightly erroneous benefit approximations necessarily occur when using the concept presented above, since its autonomous character prevents cars from having detailed knowledge about their neighbors' interest.

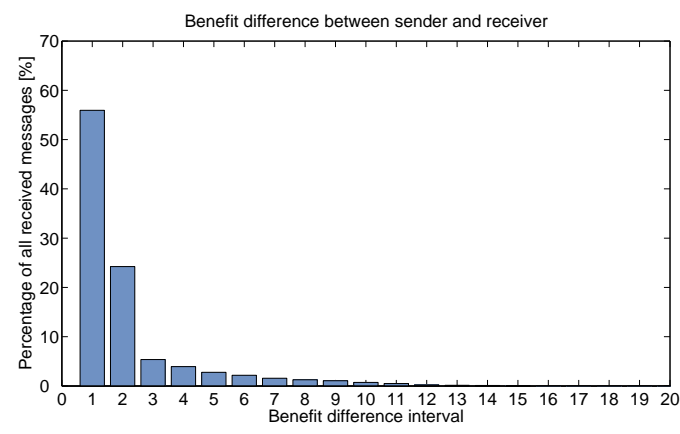

Fig. 7. Difference between senders' benefit approximations and the receivers' actual calculations

As argued above, the adaptation of the network nodes' contention windows (in the range between $C W_{\min }$ (31 slots) and $C W_{\max }(1023$ slots)) has been chosen as a major lever to realize inter-vehicle data traffic differentiation. As a consequence, defer-and backoff timers are on average longer than in case no differentiation is applied $\left(C W_{\min }\right.$ is mostly applied then). If timers are longer, the rate of data packets getting access to the medium is smaller, since the average time spent for backing off is increased. To examine the impacts on channel utilization, the global number of successfully received 


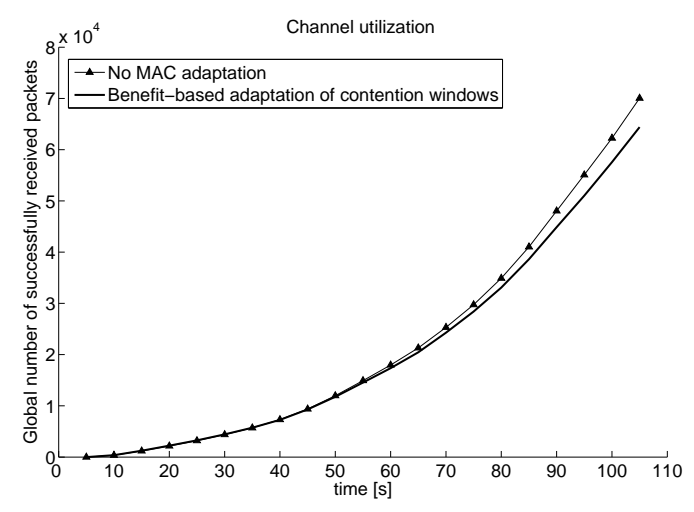

Fig. 8. Dynamic MAC adaptation slightly deteriorates channel utilization

packets in the whole network has been tracked. As one can see in Fig. 8, the modification of the MAC functionality leads to a degradation of net data throughput due to the on average timer prolongation. Note that although the net data throughput is lower due to the increased timer sizes, the global benefit is significantly higher than without traffic differentiation. The scheme is able to compensate for the decreased data throughput and considerably improve the global benefit provided to the network.

2) Simulations using the IEEE 802.11e-based architecture: Simulations showed that a IEEE 802.11e-based node architecture cannot cope with the cross-layer architecture described above. Due to the space restrictions, we pass on further details of our benchmarking analysis at this point. However, to provide a rough impression of the architecture's behavior in highly loaded VANETs, the following simulation has been performed: During 100 seconds of simulation time, packets are sorted into queues on a random basis. The number of packets transmitted has been globally tracked for each of the four priority categories. Fig. 9 shows the results of two different queue parametrization, which can be seen in table I Throughput (in number of packets dequeued per category) is in accordance with the respective category's priority. By modifying $C W_{\min }, C W_{\max }, A I F S$ and $P F$, the high priority queues' favoritism with regard to medium access can be even more enforced, where the lower priority queues are further discriminated.

\begin{tabular}{lrrrr}
\hline Parameter & Priority 1 & Priority 2 & Priority 3 & Priority 4 \\
\hline AIFS & $2 / 2$ & $2 / 2$ & $3 / 4$ & $7 / 8$ \\
$C W_{\min }$ & $7 / 2$ & $15 / 12$ & $31 / 31$ & $31 / 63$ \\
$C W_{\max }$ & $15 / 5$ & $31 / 25$ & $1023 / 1023$ & $1023 / 1023$ \\
PF & 2 & 2 & 2 & 2 \\
\hline
\end{tabular}

TABLE I

Native AND MOdified 802.11E MAC PARAMETERs (Set 2/ Set 1)

A specific scenario has been set up for evaluating the applicability of our 802.11e-based architecture in the context of crossing collision warnings. 200 vehicles are located close

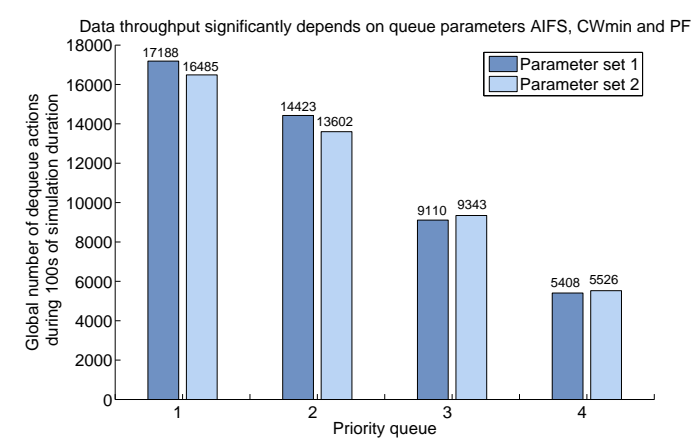

Fig. 9. Throughput of the four different packet priority queues

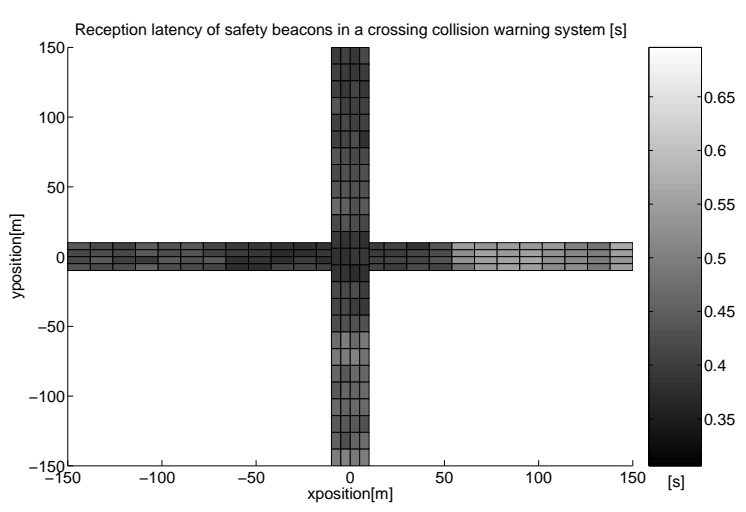

Fig. 10. Reception latency without any traffic prioritization

by a crossing which is visualized in Fig. 10 (the shaded rectangles represent the vehicles). Each road features two lanes per driving direction. All the vehicles are within one broadcast area, i.e. they are all within mutual communication range. When closer than $150 \mathrm{~m}$ to the center of the observed crossing, vehicles start to periodically (with a frequency of one second) broadcast safety beacons containing their respective geographical positions, driving directions, unique ID and time stamps. Receivers are assumed to neither store nor relay these beacons upon reception in this specific case, but extract the position of the respective senders to evaluate whether a collision is impending or not.

To optimally leverage the limited network resources and thereby increase the global benefit, one may think about introducing spatial traffic differentiation. Those beacons which are particularly important to help avoiding accidents should be favored with respect to medium access, while others may be less time-critical: In this case, the transmission priority of vehicles is determined based on the number and distance of close-by cars that drive into opposite directions. A highpriority car sorts its beacons into high priority queues to increase their reception probability.

In the first run (Fig. 11), we analyzed the latency of beacons broadcasted by each of the 200 vehicles. As a result of the spatially motivated traffic prioritization, the latency of beacons issued by cars located around the center of the crossing is 


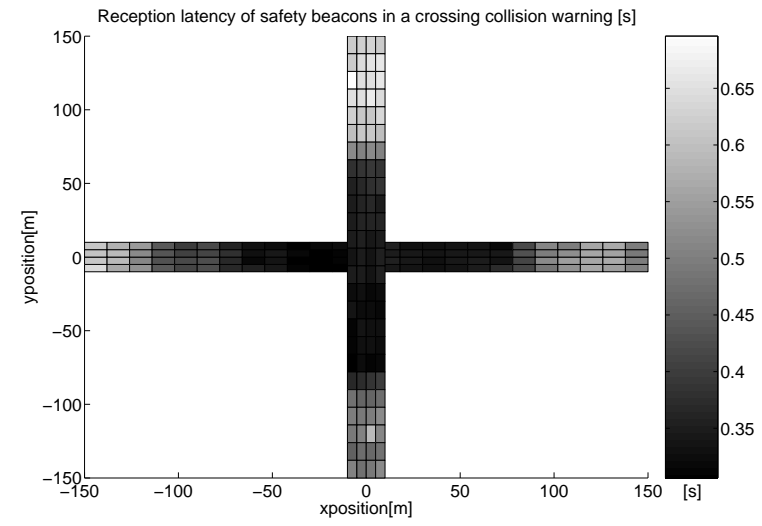

Fig. 11. Reception latency when applying traffic prioritization

rather low at the expense of those broadcasted by vehicles in the outer areas. There, the distance between cars driving into different directions is high, and thus the beacons are discriminated with regard to medium access. In Fig. 10, in contrast, no traffic differentiation is applied, leading to a rather uniformly distributed beacon latency (Reception latency in seconds is represented by the darkness of the rectangles).

\section{CONClusion}

One of the greatest challenges inherent to the deployment of VANETs is adequate information dissemination that optimally leverages resources both in sparse and dense networks. In this work, we proposed a framework for integrating many of the existing strategies that deal with reduction of superfluous transmissions or traffic differentiation in highly loaded scenarios. Network nodes thereby prioritize data according to the benefit their messages are assumed to provide for their respective neighbor nodes. As a result of this approach, the global benefit is optimized: Before transmitting any message, vehicles independently evaluate the current benefit of the packets. Based on this value, the strategy follow two main concepts. First, a local order among the messages within the send queue of each vehicle is computed. Second, the medium access strategy is adapted accordingly, favoring packets with higher expected benefit. This benefit-based message dissemination can be realized using either a proprietary cross-layer node architecture or the existing IEEE 802.11e Quality of Service standard. Extensive simulation results proved the viability of the approach and the potential comprised in using the benefit of information to schedule medium access.

\section{REFERENCES}

[1] T. Kosch, "Situationsadaptive Kommunikation in Automobilen Ad-hoc Netzen," Ph.D. dissertation, Munich University of Technology, May 2005.

[2] C. Maihöfer, W. J. Franz, and R. Eberhardt, "Stored geocast." in Proceedings of Kommunikation in Verteilten Systemen (KiVS), 2003, pp. 257-268.

[3] M. Ruffini and H.-J. Reumerman, "Distributed power control for reliable broadcast in inter-vehicle communication systems," in Proceedings of the 2nd International Workshop on Intelligent Transportation (WIT 2005), Mar. 2005
[4] C.-F. Chiasserini, E. Fasolo, R. Furiato, R. Gaeta, M. Garetto, M. Gribadou, M. Sereno, and A. Zanella, "Smart broadcast of warning messages in vehicular ad hoc networks," Workshop Interno Progetto NEWCOM (NoE), 2005.

[5] B. Williams and T. Camp, "Comparison of broadcasting techniques for mobile ad hoc networks," in Proceedings of the 3rd ACM International Symposium on Mobile Ad Hoc Networking \& Computing (MobiHoc). New York, NY, USA: ACM Press, 2002, pp. 194-205.

[6] R. Schwarz, W. Schaufelberger, L. Raymann, H. Merz, F. Zaugg, T. Kloth, and P. Farago, "Wirksamkeit und nutzen von verkehrsinformation,” Vereinigung Schweizerischer Verkehrsingenieure (SVI), Research Proposal SVI 2000/386, Aug. 2004.

[7] G. D. Veciano, "System utility functions for adaptive/ reconfigurable manets," http://www.darpa.mil/ato/solicit/CBMANET/briefs/ CBMANET_utility_metrics-deVeciana.pdf 2006.

[8] F.P.Kelly, A. Maulloo, and D.Tan, "Rate control for communication networks: shadow prices, proportional fairness and stability," in Journal of Operations Research Society, vol.49, no.3, 1998, pp. 237-252.

[9] R. J. La and V. Anantharam, "Utility-based rate control in the internet for elastic traffic," IEEE/ACM Transactions on Networking, vol. 10, no. 2, pp. $272-286$, Apr. 2002.

[10] F. Kelly, "Charging and rate control for elastic traffic," in European Transactions on Telecommunications, vol.8, no.1, Jan. 1997, pp. 33-37.

[11] M. Fazel and M. Chiang, "Nonconcave network utility maximization through sum of squares method," in Proceedings of the IEEE Control and Decision Conference, 2005.

[12] Z. Jiang, H. Mason, J. Kim, N. Shankaranarayanan, and P. Henry, "A subjective survey of user experience for data applications in future cellular wireless networks," in SAINT 2001, 2001.

[13] C.-F. Chiasserini, E. Fasolo, R. Furiato, R. Gaeta, M. Garetto, M. Gribadou, M. Sereno, and A. Zanella, "Smart broadcast of warning messages in vehicular ad hoc networks," in Workshop Interno Progetto NEWCOM (NoE), Nov. 2005.

[14] H. Füßler, J. Widmer, M. Käsemann, M. Mauve, and H. Hartenstein, "Contention-based forwarding for mobile ad-hoc networks," in Elsevier's Ad Hoc Networks, vol. 1, no.4, 2003, pp. 351-369.

[15] M. Torrent-Moreno, D. Jiang, and H. Hartenstein, "Broadcast reception rates and effects of priority access in 802.11-based vehicular ad-hoc networks," in Proceedings of the First ACM Workshop on Vehicular Ad Hoc Networks (VANET), ACM. ACM Press, Oct. 2004.

[16] P. . IEEE 802.11e/D13.0, Wireless LAN Medium Access Control (MAC) and Physical layer (PHY) specifications: Medium Access Control (MAC) Enhancements for Quality of Service (QoS), IEEE, Jan. 2005, draft Supplement to IEEE 802.11 Standard, Draft 13.0.

[17] M. Gerharz, C. de Waal, and M. Frank, "A practical view on qualityof-service support in wireless ad hoc networks," in Proceedings of the 3rd IEEE Workshop on Applications and Services in Wireless Networks $(A S W N)$, July 2003, pp. 185-196.

[18] V. Kanodia, C. Li, E. Knightly, A. Sabharwal, and B. Sadeghi, "Ordered packet scheduling in wireless ad hoc networks," in Proceedings of the 3rd ACM International Symposium on Mobile Ad Hoc Networking and Computing (MobiHoc), June 2002.

[19] U. of Southern California, "The network simulator - ns-2," http://www. isi.edu/nsnam/ns/index.html 2006

[20] M. J. Feuerstein, K. L. Blackard, T. S. Rappaport, S. Y. Seidel, and H. H. Xia, "Path loss, delay spread, and outage models as functions of antenna height for microcellular system design," IEEE Transactions on Vehicular Technology, vol. 43, no. 3, pp. 487-498, Aug. 1994.

[21] J. Peifroth, "Entwicklung und Implementierung eines FahrzeugMobilitätsmodells zur Ad Hoc Netzwerk Simulation," Master's thesis, Technische Universität München, Munich, Germany, 2002.

[22] S. Krauß, "Microscopic modelling of traffic flow: Investigation of collision free vehicle dynamics," Ph.D. dissertation, Universität Köln, Apr. 1998.

[23] S. Eichler, B. Ostermaier, C. Schroth, and T. Kosch, "Simulation of carto-car messaging: Analyzing the impact on road traffic," in Proceedings of the 13th Annual Meeting of the IEEE International Symposium on Modeling, Analysis, and Simulation of Computer and Telecommunication Systems (MASCOTS), Sept. 2005.

[24] S. Wiethölter and C. Hoene, "Design and verification of an IEEE 802.11e EDCF simulation model in ns-2.26," Telecommunication Networks Group, TU Berlin,” Technical Report, Nov. 2003. [Online]. Available: http://www.tkn.tu-berlin.de/research/802.11e_ns2 